\title{
Tratamento da xerostomia e hipossalivação em pacientes idosos
}

\author{
Treatment of xerostomy and hypossalivation in elderly patients \\ Tratamiento de xerostomia e hipossalivación en pacientes mayores
}

Recebido: 21/06/2021 | Revisado: 28/06/2021 | Aceito: 03/07/2021 | Publicado: 15/07/2021

Maria Helena Chaves de Vasconcelos Catão
ORCID: https://orcid.org/0000-0001-7681-3225
Universidade Estadual da Paraíba, Brasil
E-mail: mhelenact@ zipmail.com.br
Alana Olivia Nascimento de Souza
ORCID: https://orcid.org/0000-0002-8015-1508
Universidade Estadual da Paraíba, Brasil
E-mail: olivialanasouza @ gmail.com
Ana Clara Hecker de Carvalho
ORCID: https://orcid.org/0000-0002-0383-2238
Universidade Estadual da Paraíba
E-mail: anahecker98@ gmail.com
Arthur Cavalcanti de Oliveira
ORCID: https://orcid.org/0000-0002-2115-838X
Universidade Estadual da Paraíba
E-mail: arthuraco123@ gmail.com
Elaine Cristina Cavalcante Pinheiro
ORCID: https://orcid.org/0000-0002-7728-9119
Universidade estadual da Paraíba
E-mail:cristinapmpb@ gmail.com

\begin{abstract}
Resumo
Introdução: A população idosa sofre com diversas alterações decorrentes da senilidade, dentre elas a xerostomia e a hipossalivação. Objetivo: realizar uma revisão integrativa com o intuito de avaliar as diferentes terapêuticas descritas na literatura relacionadas ao tratamento da xerostomia e hipossalivação em idosos. Metodologia: Como estratégia de busca, foi realizada uma pesquisa bibliográfica do tipo integrativa nas bases de dados eletrônicas: PubMed, Lilacs e Web of Science, sobre o assunto, utilizando as seguintes palavras chaves: "Xerostomia", "Idosos", "Tratamento" em associação através do operador booleano AND. Foi selecionado os estudos realizados apenas em humanos entre os anos 2011 a 2021. Resultados: Entre os 94 estudos encontrados, somente 5 artigos, publicados entre os anos de 2011 a 2021, foram utilizados dos resultados. Conclusão: Após a análise dos estudos pode-se confirmar a eficácia de diversas alternativas para o tratamento da xerostomia e sua relevância científica e que a xerostomia por si só não é uma doença, mas o não tratamento traz sérias implicações odontológicas, afetando a saúde bucal e geral do paciente.
\end{abstract}

Palavras-chave: Xerostomia; Idosos; Tratamento.

\begin{abstract}
Introduction: The elderly population suffers from several changes due to senility, including xerostomia and hyposalivation. Objective: to carry out an integrative review in order to evaluate the different therapies described in the literature related to the treatment of xerostomia and hyposalivation in the elderly. Methodology: As a search strategy, an integrative bibliographic search was carried out in the electronic databases: PubMed, Lilacs and Web of Science, on the subject, using the following keywords: "Xerostomia", "Elderly", "Treatment" in association through the Boolean operator AND. Studies carried out only on humans between the years 2011 to 2021 were selected. Results: Among the 94 studies found, only 5 articles, published between the years 2011 to 2021, were used for the results. Conclusion: After analyzing the studies, it is possible to confirm the efficacy of several alternatives for the treatment of xerostomia and its scientific relevance and that xerostomia alone is not a disease, but non-treatment has serious dental implications, affecting oral health. and the patient's general.
\end{abstract}

Keywords: Xerostomia; Elderly; Treatment.

\section{Resumen}

Introduccíon: La población anciana sufre diversos cambios debidos a la senilidad, entre ellos xerostomía e hiposalivación. Objetivo: realizar una revisión integradora para evaluar las diferentes terapias descritas en la literatura relacionadas con el tratamiento de la xerostomía e hiposalivación en el anciano. Metodología: Como estrategia de búsqueda se realizó una búsqueda bibliográfica integradora en las bases de datos electrónicas: PubMed, Lilacs y Web of Science, sobre el tema, utilizando las siguientes palabras clave: "Xerostomia", "Anciano", "Tratamiento" en asociación a través de el operador booleano AND. Se seleccionaron estudios realizados solo en humanos entre los años 
2011 a 2021. Resultados: De los 94 estudios encontrados, solo 5 artículos, publicados entre los años 2011 a 2021 , se utilizaron para los resultados. Conclusión: Después de analizar los estudios, es posible confirmar la eficacia de varias alternativas para el tratamiento de la xerostomía y su relevancia científica y que la xerostomía por sí sola no es una enfermedad, pero el no tratamiento tiene serias implicaciones dentales, afectando la salud bucal. y el general del paciente.

Palabras clave: Xerostomía; Anciano; Tratamiento.

\section{Introdução}

A saliva é um dos fluidos mais essenciais do corpo. Os pacientes nunca percebem o quão importante é até que desapareça, e as coisas comuns, como comer ou falar, se tornam um problema diário (Sreebny, 2000). Dessa forma, a saliva, em um volume adequado, tem papel fundamental para uma boa qualidade de vida, uma vez que, associada a uma condição de saúde bucal satisfatória, ela protegerá o paciente de infecções da mucosa, cáries, dentre outros problemas (Diep et al., 2021).

A saliva é um fluido biológico produzido no interior das glândulas salivares, possui composição complexa enzimas, imunoglobulina A, lactoferrina, histatinas e defensinas, que fornecem atividade antimicrobiana local, atua também, como um solvente para realçar o sabor e facilitar as etapas iniciais da digestão (Baum, 1981; Ship, Nolan \& Puckett, 1995), capaz de desempenhar funções imprescindíveis à manutenção da saúde, como por exemplo: a lubrificação dos tecidos duros e moles da cavidade bucal, a manutenção da integridade da mucosa, a limpeza, a remineralização dental, a digestão, a fonação, dentre outras (Rudney, 1995). Também, ajuda a manter um pH oral neutro e fornece um reservatório de íons de cálcio e fosfato para remineralizar os dentes, protege a mucosa oral e os dentes contra substâncias nocivas; lubrifica a boca para facilitar a mastigação, deglutição e fala; e reduz o trauma do tecido (Baum, 1981; Ship et al. 1995).

A sensação de boca seca, ou xerostomia, é uma queixa comum na clínica odontológica. Segundo afirma Fleming, Craigs \& Bennett (2020) “A prevalência estimada de boca seca na população em geral está entre 21 e $27 \%$ ”. A xerostomia é definida como uma sensação subjetiva de secura bucal, que geralmente está correlacionada com diminuição do fluxo salivar (hipossalivação), enquanto a hipofunção das glândulas salivares é diagnosticada através de mensurações objetivas da redução do fluxo salivar (Fox, Van der Vem, Sonies, Weiffenbach \& Baum, 1985). No entanto, muitos pacientes apresentam sensação de boca seca com taxas de fluxo salivar normais; portanto, a xerostomia não reflete necessariamente a hipofunção da glândula salivar. A hipossalivação é definida como uma redução objetiva da taxa de fluxo salivar (Sreebny, 2000).

A boca seca tem várias consequências para a saúde bucal e afeta a qualidade de vida. A incidência de boca seca e seu impacto na saúde pública estão aumentando devido ao envelhecimento da população, aos efeitos de algumas doenças sistêmicas e ao tratamento médico e aos medicamentos comumente prescritos que reduzem a produção de saliva (Gupta, Epstein \& Srooussi, 2006).

Na população idosa particularmente a secura bucal é um problema comum, com uma ampla variação de causas que afetam importantes aspectos da vida, como a mastigação, deglutição e fala (Heydarirad \& Choopani, 2014). Ansiedade, a depressão, o comprometimento renal crônico, o diabetes mellitus, as doenças autoimunes e/ou fúngicas podem desencadear processos xerostômicos (Berti-Couto et al., 2011). Além disso, o consumo de água, hábito de fumar, higiene oral e quantidade de medicamentos utilizados são fatores associados a hipossalivação e xerostomia (Rech, Hugo, Tôrres \& Hilgert, 2019). Acreditava-se que a função salivar diminuía com a idade, mas agora é aceito que a produção de saliva e sua composição independem da idade em pessoas saudáveis (Baum, 1981; Ship et al .1995). Além disso, indivíduos mais velhos estão mais propensos ao desenvolvimento da xerostomia decorrente ao uso de medicamentos xerostômicos principalmente os diuréticos e os associados para problemas cardiovasculares (Perotto, Andrades, Paza \& Ávila, 2007; Rech \& Medeiros, 2016).

A prevalência de xerostomia aumenta com a idade e é mais comum em mulheres, aproximadamente $30 \%$ em pessoas com 65 anos ou mais (Ship, Pillemer \& Baum, 2002), provavelmente devido ao aumento da ingestão de medicamentos, devido a doenças crônicas, como hipertensão, diabetes, depressão etc., (Ship, 2002; Scully \& Bagan, 2004). Os medicamentos são a 
causa mais comum, porque a maioria dos idosos toma pelo menos um medicamento que afeta negativamente a função salivar (Schein et al., 1999). Além disso, o hábito de fumar, osofrimento psicológico, consumo deficiente de água, o cuidado de higiene oral e o tipo e a quantidade de medicamentos utilizados têm sido associados à hipossalivação e xerostomia fortemente na população (Rech et al., 2019). Dessa forma, a xerostomia e a hipossalivação podem ocasionar consequências para o idoso, uma vez que, aumenta o risco de erosão dentária, desmineralização, cárie, periodontite e afecções bucais causando, neste sentido, problemas devastadores na saúde oral (Astrom et al., 2019).

A hipossalivação é definida como uma taxa de fluxo salivar abaixo de $0,1-0,2 \mathrm{ml} / \mathrm{min}$ (não estimulada) ou $0,7 \mathrm{ml} /$ min (estimulada). A xerostomia (sensação de boca seca) é um sintoma de secura oral (Porter, Scully \& Hegarty, 2004; Scully, 2003). O tipo mais comum de xerostomia é induzida por medicamentos, pois $80 \%$ dos medicamentos mais comumente prescritos provocam redução da excreção de saliva glandular (Porter et al., 2004; Alpöz, Cankaya \& Güneri, 2015).

Uma ampla gama de terapias foi estudada para o tratamento de xerostomia, como medicamentos estimuladores da saliva (pilocarpina, fisostigmina, interferon ou cevimelina), que são eficazes para reduzir a xerostomia, mas podem ser contraindicados em pacientes com doenças respiratórias crônicas, cardiovasculares, ou doença renal (Plemons, Al-Hashimi \& Marek, 2014; Delli, Spijkervet, Kroese, Bootsma \& Vissink, 2014; Silvestre, 2004).

A xerostomia e a hipossalivação induzidas por medicamentos se tornarão cada vez mais, problemas de saúde bucal para pacientes mais velhos e geriátricos devido à provável alta prevalência de ingestão de medicamentos e polifarmácia, com um impacto negativo complexo em outros sintomas, como disfagia, incidência de cárie, desnutrição e qualidade de vida. Todos os profissionais de saúde são incentivados a investigar os sintomas de boca seca em seus pacientes, uma vez que o diagnóstico pode ser facilmente realizado na prática clínica diária (Barbe, 2018).

Portanto, a xerostomia é a sensação subjetiva de boca seca causada pela hipossalivação. É um problema comum entre os idosos. Medicamentos prescritos e sem receita, Síndrome de Sjögren e irradiação da cabeça e pescoço são as principais causas de hipossalivação em idosos. Viver com xerostomia pode ser uma experiência devastadora para o sofrimento. Por meio de diagnóstico e manejo adequados, o dentista pode ajudar a trazer algum alívio ao paciente com xerostomia (Bivona, 1998). O tratamento com enxágue oral lubrificante proporciona alívio temporário do desconforto da boca seca; no entanto, ainda não está claro como sua composição afeta os tecidos dentais mineralizados (Moynahan, Wong \& Deymier, 2021).

O objetivo deste estudo foi avaliar as diferentes terapêuticas descritas na literatura no tratamento da xerostomia e hipossalivação em idosos com vista a explorar os benefícios que os tratamentos possibilitam para a melhora da condição bucal.

\section{Metodologia}

\section{Delineamento do Estudo}

Este trabalho se trata de uma revisão de literatura integrativa qualitativa descritiva, pois contém informações relevantes na interpretação dos dados coletados, que contribuirão com os conhecimentos sobre este tema (Pereira et al., 2018).

\section{Critérios de inclusão e exclusão}

Foram incluídos artigos que abordassem o tratamento da xerostomia e/ou hipossalivação em idosos como tema principal, no período de 2011 a 2021, nos idiomas inglês e português. Os critérios de exclusão foram artigos relacionados a condições e comorbidades sistêmicas associadas, artigos de revisão, artigos não disponibilizados na íntegra e/ou estudos incompletos, estudo em animais e in vitro.

\section{Formação de informação}

Os artigos foram recuperados a partir das bases de dados: LILACS (Literatura Latino Americana e do Caribe em Ciências da Saúde), Web of Science e PubMed. O levantamento de dados ocorreu entre os meses de março e abril de 2021. 


\section{Estratégia de busca}

A estratégia de busca utilizada para a pesquisa nas bases de dados ocorreu pela utilização dos seguintes descritores e palavras-chaves: "Xerostomia", "Idosos", "Tratamento" em associação através do operador booleano AND.

\section{Resultados}

Foram encontrados 94 artigos com as palavras chaves utilizadas em associação. Desses, foram excluídos 83 por não atenderem aos critérios de inclusão do estudo. 6 artigos foram excluídos por duplicidade e apenas 5 foram incluídos na revisão integrativa. A Figura 1 descreve os estudos identificados nas bases de dados utilizadas (Pubmed, Web of Science e Lilacs) de acordo com as associações dos descritores utilizados.

Figura 1. Processo de busca, seleção e inclusão de artigos.

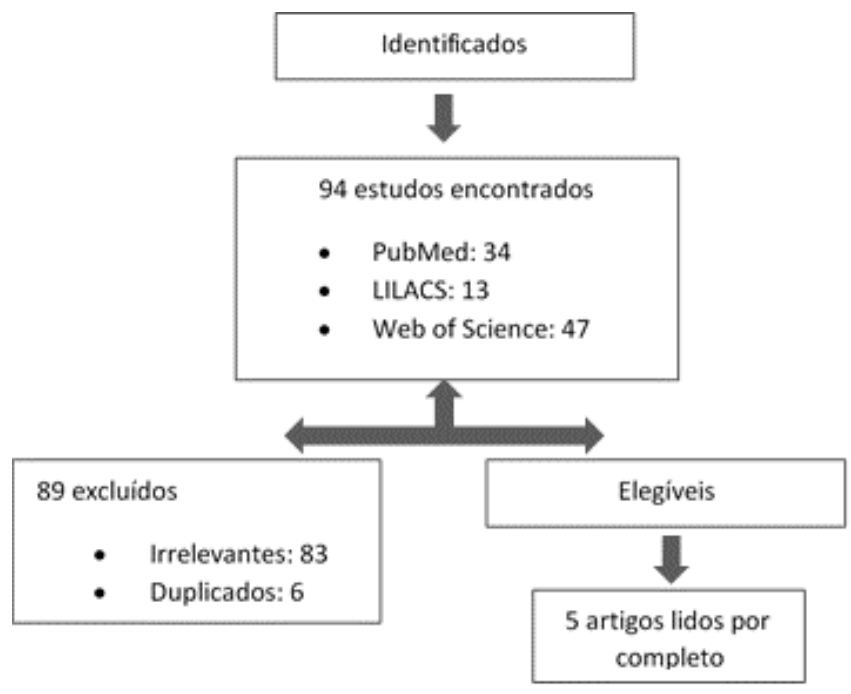

Fonte: Autores.

É importante observar a quantidade de artigos elegidos para pesquisa, pois apesar de identificados uma quantidade considerável de estudos, poucos estavam relacionados ao tema proposto, indicando que o assunto ainda é pouco abordado em pesquisas acadêmicas. A Figura 2 identifica a estratégia de busca utilizada para pesquisa e os resultados encontrados por base de dados utilizando as palavras chaves em associação. 
Figura 2. Estratégia de busca.

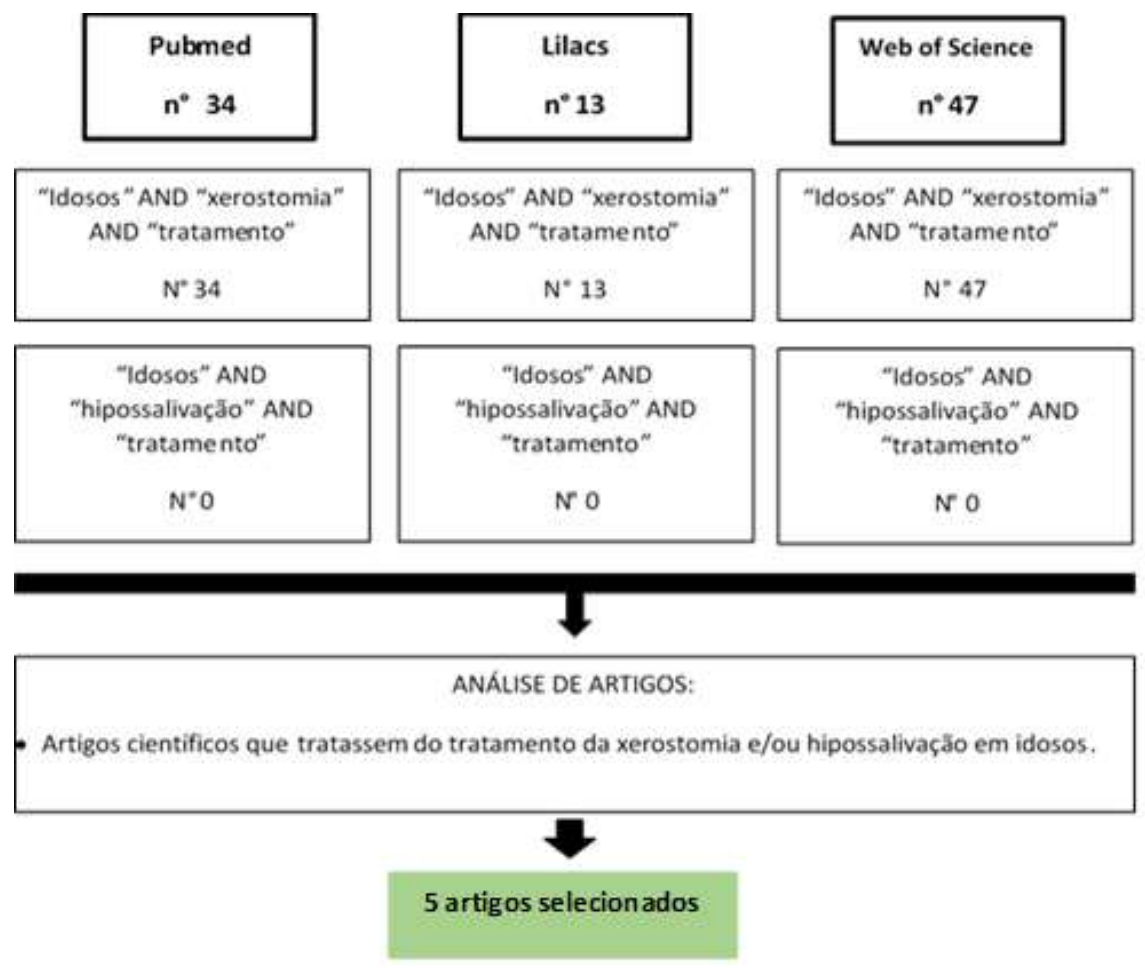

Fonte: Autores.

As associações entre descritores permitiram uma busca abrangente de artigos, da qual 5 foram eleitos conforme análise de tema.

A Tabela 1 identifica os 5 artigos utilizados na revisão integrativa, destacando: Autor/ano, título do artigo, objetivo do estudo, detalhamento do estudo ou protocolo de tratamento utilizado e conclusão.

Tabela 1. Caracterização dos estudos quanto aos objetivos, protocolos de tratamentos e conclusão.

\begin{tabular}{|c|c|c|c|c|c|}
\hline Autor/Ano & Título & Objetivos & $\begin{array}{l}\text { Detalhamento de } \\
\text { estudo }\end{array}$ & Resultados & Conclusão \\
\hline $\begin{array}{l}\text { Lončar B } \\
\qquad \text { et al. } \\
2011\end{array}$ & $\begin{array}{l}\text { The effect of } \\
\text { low-level laser } \\
\text { therapy on } \\
\text { salivar glands in } \\
\text { patients with } \\
\text { xerostomia }\end{array}$ & $\begin{array}{c}\text { Investigar o } \\
\text { efeito do lase } \\
\text { de baixa } \\
\text { potência na } \\
\text { função } \\
\text { secretora das } \\
\text { glândulas } \\
\text { salivares em } 3 \\
\text { pacientes con } \\
\text { xerostomia, } \\
\text { com idades } \\
\text { entre } 43 \text { a } 74 \\
\text { anos. }\end{array}$ & $\begin{array}{l}\text { Luz laser pulsado } \\
904 \mathrm{~nm} \text { aplicado } \\
\text { bilateralmente nas } \\
\text { glândulas salivares } \\
\text { por um período de } 10 \\
\text { dias consecutivos. A } \\
\text { densidade de energia } \\
\text { média por exposição } \\
\text { foi de } 29,5 \mathrm{~J} / \mathrm{cm}^{2}\end{array}$ & $\begin{array}{l}\text { A diferença média na } \\
\text { quantidade de } \\
\text { salivação antes e } \\
\text { após o tratamento } \\
\text { aumentou } \\
\text { linearmente do } 1^{\circ} \text { ao } \\
10^{\circ} \text { dia de terapia } \\
\text { (dQ-sal } 1 / 4 \\
0,05 \mathrm{ml} / \mathrm{min} \text { até dQ- } \\
\text { sal } 1 / 440,13 \mathrm{ml} / \mathrm{min} \text { ) }\end{array}$ & $\begin{array}{c}\text { Os efeitos da terapia a } \\
\text { laser de baixa } \\
\text { potência não são } \\
\text { apenas estimulantes, } \\
\text { mas também } \\
\text { regenerativos, uma } \\
\text { vez que a reposta } \\
\text { glandular aumentou } \\
\text { linearmente ao longo } \\
\text { do tempo. }\end{array}$ \\
\hline
\end{tabular}


Research, Society and Development, v. 10, n. 8, e42510817427, 2021

(CC BY 4.0) | ISSN 2525-3409 | DOI: http://dx.doi.org/10.33448/rsd-v10i8.17427

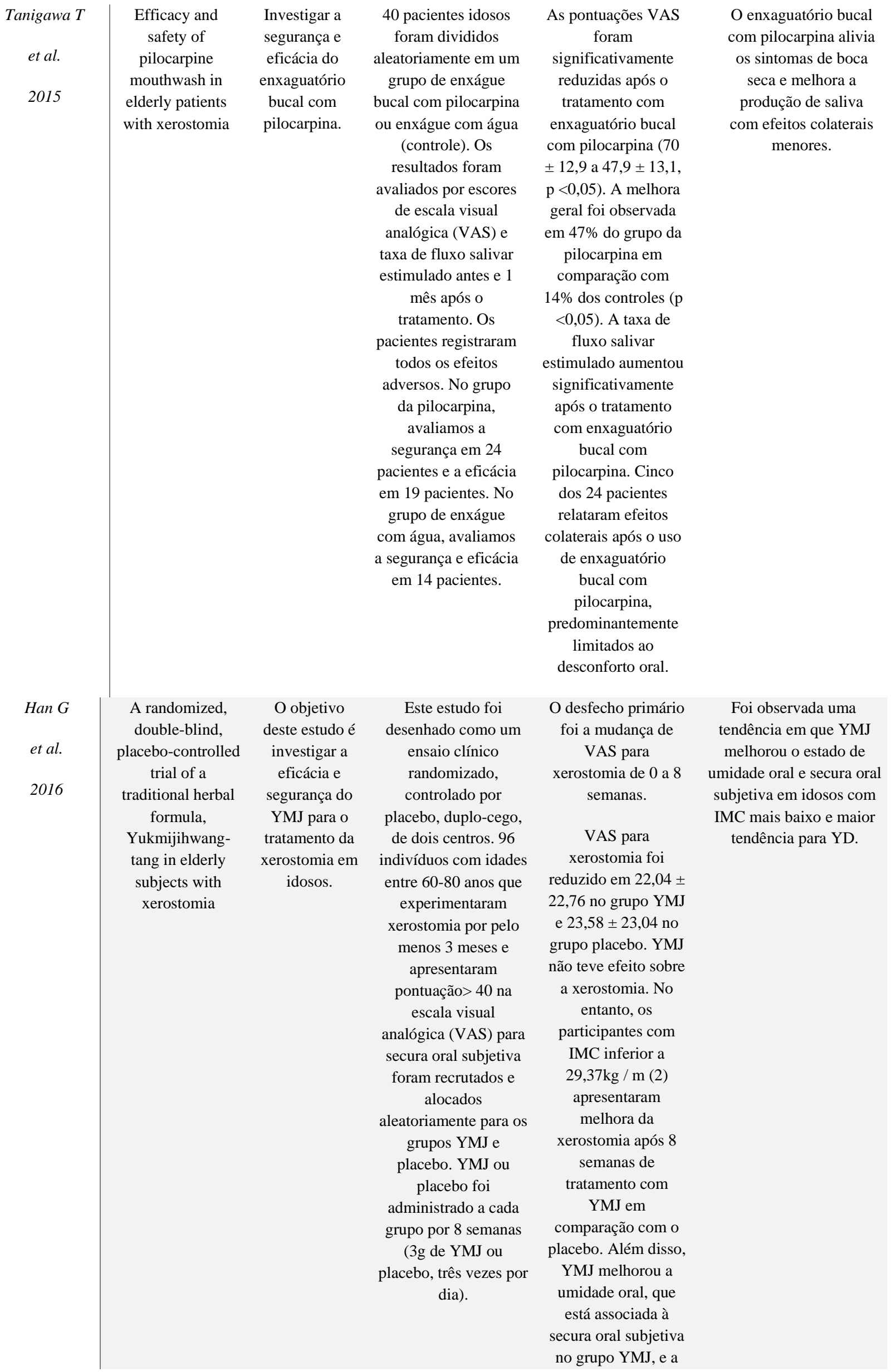




\begin{tabular}{|c|c|c|c|c|c|}
\hline & & & & $\begin{array}{l}\text { relação entre VAS } \\
\text { para xerostomia e } \\
\text { YD foi significativa. }\end{array}$ & \\
\hline $\begin{array}{l}\text { Morales- } \\
\text { Bozo I } \\
\text { et al. }\end{array}$ & $\begin{array}{l}\text { Evaluation of } \\
\text { the effectiveness } \\
\text { of a chamomile } \\
\text { (Matricaria } \\
\text { chamomilla) } \\
\text { and linseed } \\
\text { (Linum } \\
\text { usitatissimum) } \\
\text { saliva substitute } \\
\text { in the relief of } \\
\text { xerostomia in } \\
\text { elders. }\end{array}$ & $\begin{array}{c}\text { O objetivo } \\
\text { central desse } \\
\text { estudo foi } \\
\text { determinar a } \\
\text { eficácia na } \\
\text { substituição } \\
\text { da saliva por } \\
\text { um } \\
\text { composto à } \\
\text { base de } \\
\text { camomila e } \\
\text { de linhaça } \\
\text { no } \\
\text { tratamento e } \\
\text { alívio da } \\
\text { xerostomia. }\end{array}$ & $\begin{array}{c}74 \text { idosos com } \\
\text { xerostomia foram } \\
\text { selecionados para o } \\
\text { estudo. Os } \\
\text { substitutos da saliva } \\
\text { à base de ervas e da } \\
\text { saliva convencional } \\
\text { foram testados em } \\
\text { um ensaio clínico } \\
\text { duplo-cego, } \\
\text { transversal e } \\
\text { randomizado. }\end{array}$ & $\begin{array}{l}\text { Os testes clínicos } \\
\text { apresentaram nos } \\
\text { seus resultados } \\
\text { que o substituto da } \\
\text { saliva à base de } \\
\text { ervas produziu um } \\
\text { maior alívio dos } \\
\text { sintomas de boca } \\
\text { seca, sensação de } \\
\text { saliva espessa e } \\
\text { dificuldade de } \\
\text { deglutir do que o } \\
\text { convencional. }\end{array}$ & $\begin{array}{l}\text { Concluiu-se que o } \\
\text { substituto à base de } \\
\text { camomila e linhaça foi } \\
\text { muito eficaz no } \\
\text { tratamento de } \\
\text { xerostomia em pacientes } \\
\text { idosos. }\end{array}$ \\
\hline $\begin{array}{c}\text { Navarro- } \\
\text { Morante A } \\
\text { et al. } \\
2016\end{array}$ & $\begin{array}{l}\text { Natural products } \\
\text { for the } \\
\text { management of } \\
\text { xerostomia: a } \\
\text { randomized, } \\
\text { double-blinded, } \\
\text { placebo- } \\
\text { controlled } \\
\text { clinical trial }\end{array}$ & $\begin{array}{c}\text { Avaliar o } \\
\text { desempenho } \\
\text { do azeite } \\
\text { virgem } \\
\text { enriquecido } \\
\text { com } \\
\text { licopeno na } \\
\text { forma de } \\
\text { spray usado } \\
\text { para tratar } \\
\text { pacientes } \\
\text { com } \\
\text { xerostomia } \\
\text { induzida por } \\
\text { drogas, } \\
\text { comparando } \\
\text {-o com um } \\
\text { spray } \\
\text { placebo. }\end{array}$ & $\begin{array}{c}\text { Ensaio clínico } \\
\text { duplo-cego } \\
\text { randomizado incluiu } \\
60 \text { idosos com } \\
\text { xerostomia induzida } \\
\text { por drogas. O fluxo } \\
\text { salivar em repouso } \\
\text { foi medido pela } \\
\text { técnica de } \\
\text { drenagem. O } \\
\text { Inventário de } \\
\text { Xerostomia (XI) foi } \\
\text { usado para avaliar } \\
\text { os sintomas e o Oral } \\
\text { Health Impact } \\
\text { Profile } 14 \text { (OHIP- } \\
\text { 14) para avaliar a } \\
\text { qualidade de vida } \\
\text { dos pacientes. As } \\
\text { avaliações foram } \\
\text { feitas antes e depois } \\
\text { de } 12 \text { semanas da } \\
\text { aplicação do } \\
\text { produto/placebo. }\end{array}$ & $\begin{array}{l}\text { Foi observado } \\
\text { uma melhora } \\
\text { significativa na } \\
\text { qualidade de vida } \\
\text { relacionada à } \\
\text { condição oral no } \\
\text { grupo tratamento, } \\
\text { enquanto nenhuma } \\
\text { diferença } \\
\text { significativa no } \\
\text { grupo placebo. }\end{array}$ & $\begin{array}{l}\text { A aplicação tópica de } \\
\text { azeite de oliva virgem } \\
\text { enriquecido com } \\
\text { licopeno e sua } \\
\text { contraparte de placebo } \\
\text { melhorou } \\
\text { significativamente os } \\
\text { sintomas relacionados à } \\
\text { xerostomia (mas não a } \\
\text { taxa de fluxo salivar) em } \\
\text { pacientes com } \\
\text { xerostomia induzida por } \\
\text { drogas. }\end{array}$ \\
\hline
\end{tabular}

Fonte: Autores.

Na tabela, é válido observar os protocolos de tratamento seguidos e os resultados consequentes às pesquisas.

\section{Discussão}

Xerostomia é uma das complicações bucais mais frequentes encontradas na população idosa. Com o avanço da medicina está aumentando a expectativa de vida das pessoas, consequentemente, aumento da população idosa. A hipossalivação é a produção diminuída de saliva devido à hipofunção das glândulas salivares. Dentre as várias etiologias, as alterações nas glândulas salivares decorrentes do envelhecimento, o uso de medicamentos com tem poder xerostômico. 
As terapias utilizadas para minimizar os efeitos da hipossalivação e xerostomia mostraram resultados satisfatórios na melhoria da qualidade de vida, uma vez que aumentaram o fluxo salivar. Além disso, destacou-se o uso da fototerapia a laser que além do estímulo temporário e alívio paliativo dos sintomas, proporcionou, também, efeito regenerativo de função.

Em relação ao tratamento com terapia a laser de baixa intensidade, Lončar, Stipetić, Baričević (2011), realizaram um estudo utilizando luz laser Ga-As pulsado 904nm aplicado bilateralmente nas glândulas salivares em região extra oral nas glândulas parótida e submandibular, e intraoral em glândulas sublinguais, durante tratamento de 10 dias consecutivos, com tempo de exposição 120s/dia.

Os resultados obtidos afirmaram que a laserterapia resulta não apenas em um efeito estimulante temporário nas glândulas salivares, mas também atua como efeito regenerativo, restaurando sua função. O ácido cítrico, também utilizado na pesquisa como grupo controle, resulta apenas em uma resposta a curto prazo. Investigação adicional é necessária em grupos maiores de pacientes, incluindo grupos de placebo, para estabelecer se os efeitos observados são melhorias de curto prazo ou prolongadas na taxa de fluxo salivar e também para determinar a relevância e influência de certos parâmetros, como tempo de exposição, níveis médios de energia, comprimento de onda e distância da sonda. Além disso, contrariando outros estudos já realizados com o laser de baixa intensidade para tratamento de hipossalivação, não relataram efeitos colaterais ou desconforto no paciente após irradiação a laser.

Opções como o uso do azeite virgem associado ao licopeno, YMJ e enxagues bucais com pilocarpina também se mostraram eficazes ao trazer maior conforto e redução aos pacientes acometidos pela xerostomia e consequente secura bucal. $\mathrm{Na}$ avaliação quanto ao desempenho do azeite virgem aliado ao licopeno para o tratamento da xerostomia, Navarro-Morante, Wolff, Bautista e López-Jornet (2016), realizaram um ensaio duplo-cego randomizado incluindo um número de 60 (sessenta) idosos, apresentando estes, xerostomia induzida por alguma droga. Os participantes foram divididos em dois grupos, sendo um que utilizou o produto e um grupo placebo e as avaliações foram realizadas antes e após 12 semanas de aplicação.

Como resultado foi verificado uma melhora significativa na qualidade de vida do grupo que foi submetido ao uso do azeite enriquecido com licopeno, destacando que essa melhora foi observada com relação aos sintomas relacionados à xerostomia e que a taxa no fluxo salivar não sofreu alterações que se possa destacar. Esses fatores foram observados através da técnica de drenagem (para medir fluxo salivar), inventário de xerostomia (para avaliar sintomas) e Oral Health Impact (avaliar qualidade de vida).

Han et al. (2016), investigou a eficácia e segurança do Yukmijihwang-tang (YMJ) que é uma fórmula típica de ervas para tratar a síndrome de deficiência de Yin (YD), enriquecendo o humor líquido do corpo, no tratamento da xerostomia em idosos. Sendo que para esse experimento foram utilizados 96 (noventa e seis) idosos, que foram distribuídos aleatoriamente entre os grupos YMJ e placebo. O experimento foi realizado durante 8 semanas, sendo ministrado $3 \mathrm{~g}$ de $\mathrm{YMJ}$ ou placebo, três vezes ao dia.

Foi observado que o YMJ não teve efeito sobre a xerostomia, mas apresentou uma melhora no estado de umidade oral e secura subjetiva, para pacientes com o IMC baixo (inferior a 23,37kg) e que a escala visual analógica (VAS) para secura oral subjetiva sofreu mudanças, com redução de valores nos grupos YMJ e placebo.

Em relação a eficácia do enxaguatório bucal com pilocarpina, Tanigawa et al. (2015), o estudo foi realizado com 40 idosos divididos entre os grupos com enxágue bucal com pilocarpina e enxague com água (grupo controle). Para avaliar os resultados foram utilizados a escala visual analógica (VAS) e a taxa de fluxo salivar estimulado, sendo coletados antes e um mês após o tratamento.

As particularidades observadas com o estudo foram: uma melhora geral em $47 \%$ do grupo que fez uso da pilocarpina, comparado a $14 \%$ no grupo controle; um aumento na taxa de fluxo salivar no grupo pilocarpina; alívio dos sintomas de boca seca e melhora na produção salivar e relatos de efeitos colaterais relacionados com desconforto oral, em cinco dos 24 
participantes do grupo pilocarpina. Para os efeitos adversos foram apresentadas duas explicações possíveis, sendo uma delas, o estímulo das glândulas salivares devido ao contato direto com a pilocarpina, ocasionando uma sensação de desconforto na boca e outra possível explicação é que as mucosas orais desses pacientes tenham hipersensibilidade causada por boca seca prolongada. Este estudo possui limitações, como por exemplo, não ter sido possível investigar os efeitos da concentração de pilocarpina sobre sintomas.

Portanto, a partir dessa discussão é fundamental priorizar a importância da pesquisa já realizada e sua continuidade sobre os mais variados métodos de alívio da xerostomia, incluindo meios medicamentosos, naturais ou químicos, assim como os não medicamentosos, garantindo uma possibilidade de melhoria de qualidade de vida para a população acometida.

\section{Conclusão}

Com base nos estudos realizados na literatura consultada, concluiu-se que há poucas alternativas referentes à abordagem do tratamento da xerostomia, que ocorre mais em idosos e é induzida pelo uso de medicamentos ou como sintoma secundário de alguma síndrome ou doença pré-existente, havendo uma estreita relação entre hipossalivação e determinados medicamentos e respectivas patologias sistêmicas.

Ressalta-se a necessidade de realização de mais estudos clínicos com pacientes idosos, a fim de ampliar a possibilidade de tratamentos.

\section{Referências}

Alpöz, E., Cankaya, H., Güneri, P., Epstein, J. B., Boyacıoglu, H., Kabasakal, Y., \& Ocakci, P. T. (2015). Impact of Buccothermâon xerostomia: a single blind study. Special Care Dentistry, 35(1), 1-7.

Åstrøm A. N., Lie S. A., Ekback G., Gülcan F., Ordell S. (2019). Self-reported dry mouth among ageing people: a longitudinal, cross-national study. European Journal of Oral Sciences. 127(2), 130-138.

Barbe, A.G. (2018). Medication-Induced Xerostomia and Hyposalivation in the Elderly: Culprits, Complications, and Management. Drugs \& Aging, 35(10), $877-885$.

Baum B. J. (1981). Evaluation of stimulated parotid saliva flow rate in different age groups. Journal of dental research, 60(7), $1292-1296$.

Berti-Couto, S. de A., Couto-Souza, P. H., Jacobs, R., Nackaerts, O Rubira-Bullen, I. R. F., Westphalen, F. H., Moysés, S. J., Ignácio, S. A., Costa, M. B. da, \& Tolazzi, A. L. (2012). Clinical diagnosis of hyposalivation in hospitalized patients. Journal of Applied Oral Science. 20(2), 157-161

Bivona P.L.(1998). Xerostomia. A common problem among the elderly. New York State Dental Journal, 64(6), 46-52.

Brunetti-Montenegro, F. L., \& Marchini, L. (2013). Odontogeriatria: uma visão gerontológica. Elsevier.

Delli, K., Spijkervet, F. K., Kroese, F. G., Bootsma, H., \& Vissink, A. (2014). Xerostomia. Monographs in Oral Science, 24,109-25.

Diep, M. T., Jensen, J. L., Skudutyte-Rysstad, R., Young, A.,Sødal, A. T. T., Petrovski, B. É., Hove, L. H. (2021). Xerostomia and hyposalivation among a 65yr-old population living in Oslo, Norway. European Journal of Oral Science., 129(1), 1-11.

Fleming, M, Craigs, C. L., Bennett, M. I. (2020). Palliative care assessment of dry mouth: what matters most to patients with advanced disease?. Supportive Care in Cancer, 28(1), 1121-1129.

Fox, P. C., Van der Ven, P. F., Sonies, B. C., Weiffenbach, J. M., \& Baum, B. J. (1985). Xerostomia: evaluation of a symptom with increasing significance. The Journal of the American Dental Association, 110(4),519-25.

Gupta A., Epstein J.B., \& Sroussi H. (2006). Hyposalivation in elderly patients. Journal of the Canadian Dental Association, 72(9), 841-846.

Han, G., Ko, S. J., Kim, J., Oh, J. Y., Park, J. W., \& Kim, J. (2016). A randomized, double-blind, placebo-controlled trial of a traditional herbal formula, Yukmijihwang-tang in elderly subjects with xerostomia. Journal of ethnopharmacology. 182, 160-169.

Heydarirad, G., \& Choopani, R. (2015). "Dry mouth" from the perspective of traditional Persian medicine and comparison with current management. Journal of evidence-based complementary \& alternative medicine. 20(2), 137-142.

Lončar, B.; Stipetic, M.M.; Barcevic, M., \& Risovic, D. (2011). The Effect of Low-Level Laser Therapy on Salivary Glands in Patients with Xerostomia Photomedicine and Laser Surgery, 29(3), 171-175.

Moraes, C. V., Albuquerque, L. C., \& Chevitarese, L. (2017). A importância da Odontogeriatria para a oferta de cuidados bucais em idosos. Revista Rede de Cuidados em Saúde. 10(1), 1-9. 
Research, Society and Development, v. 10, n. 8, e42510817427, 2021

(CC BY 4.0) | ISSN 2525-3409 | DOI: http://dx.doi.org/10.33448/rsd-v10i8.17427

Moynahan, M. M., Wong, S. L., \& Deymier, A. C. (2021). Beyond dissolution: Xerostomia rinses affect composition and structure of biomimetic dental mineral in vitro. PloS one, 16(4), 1-20

Navarro Morante, A., Wolff, A., Bautista Mendoza, G. R., \& López-Jornet, P. (2017). Natural products for the management of xerostomia: a randomized, double-blinded, placebo-controlled clinical trial. Journal of oral pathology \& medicine: official publication of the International Association of Oral Pathologists and the American Academy of Oral Pathology. 46(2), $154-160$.

Pereira, A. S., Shitsuka, D. M., Parreira, F. J., \& Shitisuka A. R. (2018). Metodologia da pesquisa científica. Santa Maria, RS, Ed.UAB/NTE/UFSM.

Perotto, J. H., Andrades, K. M. R., Paza, A. O., \& Ávila, L. F. de C.(2007). Prevalência da xerostomia relacionada à medicação nos pacientes atendidos na Área de Odontologia da UNIVILLE. Revista Sul-Brasileira de Odontologia, 4(2), 16-19.

Plemons, J. M., Al-hashimi, I., Marek, C. L. (2014). Managing xerostomia and salivary gland hypofunction: executive summary of a report from the American Dental Association Council on Scientific Affairs. The American Journal of the Dental Association, 145(8),867-73.

Porter, S. R., Scully, C., \& Hegarty, A. M. (2004). An update of the etiology and management of xerostomia. Oral surgery, oral medicine, oral pathology, oral radiology, and endodontics, $97(1), 28-46$.

Rech, C. B. (2016). Xerostomia associada ao uso de medicamentos em idosos. Journal Oral Invest, 5(1), 13-18.

Rech, R. S., Hugo, F. N., Tôrres, L., \& Hilgert, J. B. (2019). Factors associated with hyposalivation and xerostomia in older persons in South Brazil. Gerodontology, 36(4), 338-344.

Rudney, J. D. (1995). Does Variability in Salivary Protein Concentrations Influence Oral Microbial Ecology and Oral Health? Critical Reviews in Oral Biology \& Medicine, 6(4), 343-367.

Schein, O. D., Hochberg, M. C., Muñoz, B., Tielsch, J. M., Bandeen-Roche, K., Provost, T., Anhalt, G. J., \& West, S. (1999). Dry eye and dry mouth in the elderly: a population-based assessment. Archives of internal medicine, 159(12), 1359-1363.

Scully C. (2003). Drugs effects on salivary glands: dry mouth. Oral Disease, 9, 165-176.

Scully, C., \& Bagan, J.-V. (2004). Adverse Drug Reactions in the Orofacial Region. Critical Reviews in Oral Biology \& Medicine, 15(4), 221-239.

Ship J. A. (2002). Diagnosing, managing, and preventing salivary gland disorders. Oral diseases, 8(2), 77-89.

Ship, J. A., Nolan, N. E., \& Puckett, S. A. (1995). Longitudinal analysis of parotid and submandibular salivary flow rates in healthy, different-aged adults. The journals of gerontology. Series A, Biological sciences and medical sciences, 50(5), 285-289.

Ship, J. A., Pillemer, S. R., \& Baum, B. J. (2002). Xerostomia and the geriatric patient. Journal of the American Geriatrics Society, 50(3), 535-543.

Silvestre-Donat, F. J., Miralles-Jordá, L., \& Martínez-Mihi, V. (2004). Protocol for the clinical management of dry mouth. Medicina oral: organo oficial de la Sociedad Espanola de Medicina Oral y de la Academia Iberoamericana de Patologia y Medicina Bucal, 9(4), $273-279$.

Sreebny L. M. (2000). Saliva in health and disease: an appraisal and update. International Dental Journal, 50(3), 140-161.

Tanigawa, T., Yamashita, J., Sato, T., Shinohara, A., Shibata, R., Ueda, H., \& Sasaki, H. (2015). Efficacy and safety of pilocarpine mouthwash in elderly patients with xerostomia. Special care in dentistry: official publication of the American Association of Hospital Dentists, the Academy of Dentistry for the Handicapped, and the American Society for Geriatric Dentistry, 35(4), 164-169. 